eCommons@AKU

THE AGA KHAN UNIVERSITY

Department of Medicine

Department of Medicine

January 2009

\title{
PO10.19 clinical spectrum of pseudo seizures at a tertiary care hospital Karachi: a video EEG based study
}

Nadeem Ahmed Memon

Aga Khan University

Bhojo A. Khealani

Aga Khan University

Fazal Karim

Aga Khan University

Mustafa Khan

Aga Khan University

Nadir Ali Syed

Aga Khan University

See next page for additional authors

Follow this and additional works at: https://ecommons.aku.edu/pakistan_fhs_mc_med_med Part of the Neurology Commons

\section{Recommended Citation}

Memon, N., Khealani, B., Karim, F., Khan, M., Syed, N., Shafqat, S. (2009). PO10.19 clinical spectrum of pseudo seizures at a tertiary care hospital Karachi: a video EEG based study. Clinical Neurophysiology, 120(1), PO10.

Available at: https://ecommons.aku.edu/pakistan_fhs_mc_med_med/544 
Authors

Nadeem Ahmed Memon, Bhojo A. Khealani, Fazal Karim, Mustafa Khan, Nadir Ali Syed, and Saad Shafqat 
P010.17

The Effect of Prophylactic Therapy with Valproate Sodium in Japanese Children with Cyclic Vomiting Syndrome

Sono Kaneko*, Toshiyuki Hikita, Hiroko Kodama, Natsue Nakamoto, Fumiaya Kaga, Kaori Amakata, Kaori Ogita, Yasushi Fujii, Yukishige Yanagawa

Dept. of Pediatrics, Teikyo University School of Medicine, Japan

E-mail address: sono-1214-yosm@tcn-catv.ne.jp

Background: Cyclic vomiting syndrome (CVS) is characterized by recurrent, stereotypic episodes of incapacitating nausea, vomiting, and other symptoms, separated by intervals of comparative wellness. The second edition of the International Headache Classification classifies CVS as the subgroup of childhood periodic syndromes, which are common precursors of migraine. CVS may respond to migrainedirected prophylactic agents such as beta-blockers, amitriptyline, and cyproheptadine. Since 1988, valproate has been prescribed for migraine prophylaxis. The aim of this therapeutic trial was to evaluate the use of valproate, an antimigraine prophylactic drug, for the prophylactic management of CVS in children. We used prophylactic therapy in patients in whom the episodes of CVS occurred more-than-once a month and/or who particularly has severe and disabling episodes.

Methods: The nature of the trial and the possible side effects of the drug were explained to the prospective participants, i.e., parents as well as patients. After receiving an informed consent, 13 children diagnosed with severe CVS were enrolled in the study. Prophylactic therapy consisted of valproate administered at a dose of $10-40 \mathrm{mg}$. All patients underwent diagnostic tests to rule out organic causes of their symptoms. Vomiting was severe enough in all patients to cause dehydration requiring hospitalization for intravenous rehydration. Electroencephalogram (EEG) showed spikes or spike-wave complexes in two patients, and normal EEG in the remaining patients. Nine of the 13 patients did not respond to medical therapies such as propranolol, amitriptyline, cyproheptadine, phenobarbital, phenytoin, and carbamazepine. Three patients required combination therapy with valproate and phenobarbital.

Results: Of the 13 patients, two showed complete resolution of their symptoms; nine showed marked improvement, which was evidenced by a low number of attacks with reduced severity; and two failed to respond to valproate therapy. Four patients experienced relapse with a decreased dosage of valproate. Side effects associated with long-term valproate administration were not observed, with the exception of mild liver dysfunction.

Conclusions: Valproate appears to be effective for the prophylactic management of severe CVS, with $85 \%$ of all patients achieving a reduction in the frequency of attacks.

P010.18

Prognostic Impact of Hippocampal Sclerosis in Central Nervous System Infection-Related Epilepsy

Soochul Park ${ }^{1 *}$, Joo-Hyun Seo ${ }^{1}$, Bosuk Park ${ }^{1}$, Moon Kyu Lee ${ }^{1}$, Yeung-Yeob Kim²

${ }^{1}$ Dept. of Neurology, Yonsei University, College of Medicine, Korea,

${ }^{2}$ Diagnostic Radiology, Yonsei University, College of Medicine, Korea

E-mail address: scpark@yumc.yonsei.ac.kr

Background: Central nervous system (CNS) commonly resulted in encephalomalacia (ECM) and/or hippocampal sclerosis (HS), which was relevant lesion of chronic epilepsy. The significance of the prognostic impact of HS in CNS infection-related epilepsy has not been studied. We tried to study the prognostic impact of HS irrespective of ECM in central nervous system infection-related epilepsy.

Methods: Total 102 epilepsy patients with antecedent history of meningoencephalitis were recruited. Those were divided into as followings; 35 patients with unilateral isolated HS as group A. 24 patients with ECM in addition to unilateral $\mathrm{HS}$ as group B, 22 patients with no lesion on brain MRI as group C, 21 patients with ECM lesion only as group D. Prognostic impact depending on the presence of HS was compared between group A and C. Comparison was also done between group B and D. Finally, analysis depending on the presence of HS, irrespective of ECM, was done. Age at onset of epilepsy, age of infection, clinical variables with neurologic status, clinical characteristics of seizure, and semiologic features of temporal lobe epilepsy (TLE) were analyzed. Prognosis with a follow-up duration of at least two years was analyzed. Results: Comparison of clinical variables between group $A$ and $C$ showed that semiological features of TLE were significantly prominent in group A, but 2' GTC and nocturnal dominance were more in group C. In the analysis depending on the presence of HS irrespective of ECM, semiological features of TLE were statistically prominent in group with HS but 2' GTC was more in group without HS. There was no statistically significant difference in prognosis between any comparisons.

Conclusions: In general consideration of the poor prognostic impact of HS in epilepsy, the presence of HS in CNS infection-related epilepsy irrespective of the underlying ECM did not make statistically significant impact on the prognosis of CNS infection-related epilepsy.

P010.19

Clinical Spectrum of Pseudo Seizures at a Tertiary Care Hospital Karachi: A Video EEG Based Study

Nadeem Ahmed Memon ${ }^{1,2}$ *, Bhojo A. Khealani1,2, Fazal Karim ${ }^{1}$, Mustafa Khan ${ }^{1}$, Nadir Ali Syed ${ }^{2}$, Saad Shafqat ${ }^{1,2}$

${ }^{1}$ Clinical Neurophysiology Dept., The Aga Khan University Karachi, Pakistan, ${ }^{2}$ Section of Neurology, Dept. of Medicine, The Aga Khan University Karachi, Pakistan

E-mail address: nadeem.memon@aku.edu

Background: Nonepileptic seizures are involuntary episodes of movement, sensation, or behaviors (e.g., vocalization, crying and other expressions of emotion), that do not result from abnormal cortical discharges. The events typically occur in teenage patients with anxiety and affective disorders. Psychotherapy, behavioral therapy, drugs, hypnosis and placebo are mainstay of treatment. There is no published data on clinical characteristics of pseudo-seizures from Pakistan. The objective of our study is to describe the clinical characteristics of pseudoseizures in a tertiary care hospital in Karachi, Pakistan.

Methods: This is a descriptive study. Patients with pseudo-seizures were identified from departmental Video EEG monitoring register, over a period of three years (2004-2006). Their neurophysiologic and video recording data was reviewed and their demographic, clinical and neurophysiologic data was recorded and analyzed. SPSS version 15.0 was used for analysis. Results: A total of 132 patients underwent video EEG during the period and 51 (38\%) had pseudo-seizures. Their mean age was $26.7 \pm 15.3$ years. Thirty-two $(63 \%)$ were female and $19(37 \%)$ were male. Twelve $(23.5 \%)$ patients were known epileptics. Median recording time was 24 (range; $0.33-55$ ) hours and median number of events was 3 (range; 1-14). Limb movements were most common manifestation $(67 \%)$ followed by muteness with no response to verbal commands (49\%), behavioral symptoms (35.5\%), ocular findings i.e. eye blinking and closure $(25.5 \%)$, depressed conscious level (13.7\%), headache (10\%) and Gl symptoms $(10 \%)$. Six $(11.8 \%)$ patients experienced epileptic seizures, in addition to pseudo-seizures.

Conclusions: Pseudo-seizures are common in patients referred for video EEG monitoring. It is more common in young women. Limb movements, muteness and behavioral symptoms are most common manifestations. The pseudo-seizures and epileptic seizures may coexist in the same patient. 